EDITORIAL

\title{
Optimal therapeutic strategies in the setting of post-infarct no reflow: the need for a pathogenetic classification
}

\section{Galiuto}

.

Although no reflow is clearly associated with a poor prognosis, little guidance is available on the treatment of this phenomenon. The ideal therapeutic approach comprises drugs with multiple actions on the microvascular damage produced by ischaemia/reperfusion, as well as knowledge of the time course of events in order to optimise the timing of administration

Correspondence to: Leonarda Galiuto, MD, $\mathrm{PhD}$, Institute of Cardiology, Catholic University of the Sacred Heart, Policlinico A. Gemelli, Largo A. Gemelli, 8, 00136 Rome, Italy; lgaliuto@rm.unicatt.it
$\mathrm{E}$ xtensively studied both in the experimental and clinical setting, the no reflow phenomenon is clearly associated with unfavourable clinical outcome and prognosis. ${ }^{1}$ Despite the large body of evidence on the efficacy of different possible therapeutic approaches, clear guidelines on the treatment of no reflow have not been given; thus, the phenomenon is not consistently and uniformly treated in clinical practice. A possible reason for this apparent paradox may reside in the confusion generated by the multifactorial pathogenesis of no reflow, with consequent difficulty in the design of an adequate therapeutic strategy. Thus, there is a need for a pathogenetic classification of the phenomenon as an important premise for targeted forms of treatment.

\section{STRUCTURAL AND FUNCTIONAL NO REFLOW: A NOVEL PATHOGENETIC CLASSIFICATION}

By definition, no reflow is the inability to reperfuse a myocardial region after prolonged ischaemia, despite reopening of the infarct related artery. Based on morphological and functional studies, ${ }^{23}$ the phenomenon may be classified into two different forms: structural and functional. In structural no reflow, microvessels confined within necrotic myocardium exhibit irreversible damage of the cellular components of their wall; in functional no reflow, patency of anatomically intact microvessels is compromised because of spasm and/or microembolisation. The need for a classification of the no reflow phenomenon comes from the observation that the pathogenesis, time course, functional implications, and possible therapeutic approach of structural and functional no reflow are different. Studied in experimental preparations, structural no reflow is the result of the loss of integrity of endothelial cells, with microvascular obstruction caused by fibrin thrombi and/or compression from surrounding necrotic tissue. ${ }^{2}$ Because of massive morphological changes in microcirculation, the damage is necessarily irreversible and,
Heart 2004;90:123-125. doi: 10.1136/hrt.2003.020800

furthermore, it is confined within myocardial tissue that is also irreversibly damaged by the ischaemic injury.

Functional no reflow, on the other hand, has been primarily observed in humans since its pathogenesis relates more closely to events occurring in an individual undergoing an acute, reperfused, myocardial infarction with consequent activation of the neurohumoral system and peripheral dissemination of thrombus debris. ${ }^{3}$ Methods to detect possible microvascular obstruction to flow, such as coronary angiography, myocardial contrast echocardiography, and magnetic resonance imaging, have enabled the dynamic nature of the no reflow phenomenon to be determined.

\section{THERAPEUTIC APPROACH TO STRUCTURAL NO REFLOW}

The extension of structural no reflow is directly determined by the duration and severity of the ischaemic injury. Although ischaemia is the main determinant of myocardial and microvascular damage, the re-establishment of coronary flow carries a large additional injury attributed to a rapid, almost explosive, increase in tissue water, oxygen free radicals, $\mathrm{Na}^{+}, \mathrm{Cl}^{-}$and $\mathrm{Ca}^{2+}$, producing pronounced myocardial and endothelial cell swelling and contraction band necrosis, followed by further structural microvascular disruption. Furthermore, blood cells reoccupy the post-ischaemic microvascular compartment, resulting in packing of red blood cells that lose their flexibility within ischaemic tissue and plugging of leucocytes because of cell activation with enhanced adhesion properties. ${ }^{2}$ However, if sufficient adenosine triphosphate (ATP) remains available to preserve membrane ultrastructure and calcium homeostasis during ischaemia, overloading with calcium will not occur on reperfusion and the mitochondria will not accumulate $\mathrm{Ca}^{2+}$, ensuring their functional survival.

The therapeutic approach to this form of no reflow requires both reopening of the infarct related artery in the shortest possible time, and limitation of the severity of the ischaemic burden by reduction of oxygen consumption with $\beta$ blockers and calcium channel blockers. Nicorandil, a hybrid of ATP sensitive $\mathrm{K}^{+}$channel opener-tested by Ikeda and colleagues in a study in patients undergoing primary percutaneous coronary intervention (PCI) and published in this issue of Heart-might help by reducing pre- and afterload with consequent reduction in oxygen requirements. ${ }^{4}$ 
Furthermore, nicorandil, along with calcium antagonists, protects against reperfusion induced calcium overload. Indeed, ATP sensitive $\mathrm{K}^{+}$channel opening by nicorandil shortens the duration of action potential of ischaemic myocardium, thus inhibiting voltage dependent $\mathrm{Ca}^{2+}$ channels and $\mathrm{Na}^{+} / \mathrm{Ca}^{2+}$ exchange with consequent cardioprotection. Finally, both calcium antagonists and nicorandil have been shown to provide protection against oxygen free radicals, and to possess neutrophil modulating properties. ${ }^{5}$ Thus, if sufficient ATP remains available to preserve cell membrane ultrastructure and calcium homeostasis during ischaemia, overloading with calcium will not occur on reperfusion and the mitochondria will not accumulate calcium, ensuring their functional survival. On the other hand, if the cell membrane is disrupted, calcium antagonists and even nicorandil are unlikely to have a beneficial role.

\section{THERAPEUTIC APPROACH TO FUNCTIONAL NO REFLOW}

Functional no reflow is the result of abrupt microvascular obstruction characterised by a dynamic time course and potential reversibility. ${ }^{4}$ We have recently observed that in about $50 \%$ of post-infarct no reflow patients, the phenomenon is reversible within the first month. ${ }^{6}$ In these patients, left ventricular volumes did not dilate, as opposed to progressive dilatation in sustained no reflow patients, despite lack of significant improvement in regional left ventricular function. The pathogenesis of this form of reversible no reflow remains to be fully elucidated. Due to their reversibility, microvascular spasm and microembolisation are two possible determinants of temporary obstruction of microcirculation after prolonged ischaemia.

The presence of microvascular spasm has been indirectly shown by the improvement in post-ischaemic flow produced by pharmacological intervention to antagonise the effect of post-ischaemic vasoconstriction, such as calcium channel blockers. $^{7}$ Since nicorandil acts as a vasodilator of small coronary arteries, the early improvement of left ventricular function observed by Ikeda and colleagues ${ }^{4} 30$ minutes after reperfusion might be caused by prevention of small vessel spasm. ${ }^{4}$ Accordingly, Ikeda and colleagues ${ }^{4}$ observed an improvement in systolic coronary flow velocity measured by Doppler catheter immediately after PCI with nicorandil, but not with glyceryl trinitrate, probably because ATP sensitive $\mathrm{K}^{+}$channel openers, but not nitrates, dilate arterioles $<100 \mu \mathrm{m}$ in diameter.

The role of vasoconstriction in the genesis of reversible no reflow is confirmed by the beneficial effect demonstrated with endothelin antagonists in an experimental model of ischaemia reperfusion, ${ }^{8}$ and by the improvement of left ventricular dysfunction with $\alpha$ adrenergic blockade and by adrenaline (epinephrine) treatment in post-infarct patients. ${ }^{910}$

Distal embolisation has been effectively prevented by Filter Wire-Ex, a distal embolic protection device, with beneficial effect on markers of myocardial reperfusion (corrected TIMI frame count, grade 3 myocardial blush, early ST segment elevation resolution, peak creatine kinase release) and on left ventricular wall motion score index and ejection fraction. ${ }^{11}$ Interestingly, in the PAMI (primary angioplasty in myocardial infarction) trial, ${ }^{12}$ stenting was followed by decreased post-procedural flow as compared to balloon angioplasty, suggesting that the additional squeezing of plaque material produced by stent placement is followed by significant distal embolisation of debris, producing plugging of distal microvessels and consequent reduction in coronary reflow.

Furthermore, abciximab treatment substantially augments microvascular perfusion and improves myocardial performance, most likely due to limited platelet aggregation in the microcirculatory zone recipient of embolic material. ${ }^{13}$

Lastly, the individual response to microembolisation may be further modulated by anti-inflammatory drugs. In fact, inflammation may have a role not only in determining the friability of atheromatous lesion, but also in determining the response to embolisation at the microvascular level. Nicorandil might be more effective than verapamil in the prevention of no reflow after primary PCI, possibly because of an anti-inflammatory effect, such as the inhibition of neutrophils. ${ }^{14}$

\section{ADEQUATE TREATMENT FOR THE INDIVIDUAL PATIENT}

The ideal therapeutic approach of such a multifactorial phenomenon as no reflow comprises drugs with multiple actions on the microvascular damage produced by ischaemia/ reperfusion, and requires knowledge of the time course of events in order to optimise the timing of administration. In this respect, the study by Ikeda and colleagues appears adequately designed, since nicorandil has a large spectrum of effects which might counteract several mechanisms of both structural and functional no reflow. ${ }^{4}$

However, the therapeutic approach to no reflow should take into account the prevailing mechanism operating in the individual patient. Notably, concomitant diseases may influence the occurrence and natural history of the no reflow phenomenon along with the response to treatment. For example, hyperglycaemia is associated with a larger prevalence of no reflow, possibly due to the damage of the coronary microcirculation. ${ }^{15}$ Similarly, hypercholesterolaemia is associated with a $40 \%$ increase in no reflow area, due to blockade of $\mathrm{K}^{+}$channels, and treatment with nicorandil may reduce the extent of this damage. ${ }^{16}$

\section{REFERENCES}

1 Ito $\mathrm{H}$, Maruyama A, Iwakura K, et al. Clinical implications of the "no-reflow" phenomenon. A predictor of complications and left ventricular remodeling in reperfused anterior wall myocardial infarction. Circulation 1996;93:223-8.

2 Kloner RA, Ganote CE, Jenning RB. The "no-reflow" phenomenon after temporary coronary occlusion in dogs. J Clin Invest 1974;54:1496-508.

3 Wilson RF, Laxson DD, Lesser JR, et al. Intense microvascular constriction after angioplasty of acute thrombotic coronary arterial lesions. Lancet 1989;i:807-11.

4 Ikeda N, Yasu T, Kubo N, et al. Nicorandil versus isosorbide dinitrate as adjunctive treatment to direct balloon angioplasty in acute myocardial infarction. Heart 2004;90:181-5

5 Taniyama $Y$, Ito $H$, Iwakura $K$, et al. Beneficial effect of intracoronary verapamil on microvascular and myocardial salvage in patients with acute myocardial infarction. J Am Coll Cardiol 1997;30:1193-9.

6 Galiuto L, Lombardo A, Maseri A, et al. Temporal evolution and functional outcome of no-reflow: sustained and spontaneously reversible patterns following successful coronary recanalization. Heart 2003;89:731-7.

7 Piana RN, Paik GY, Moscucci M, et al. Incidence and treatment of "no-reflow" after percutaneous coronary intervention. Circulation 1994;89:2514-8.

8 Galiuto L, DeMaria AN, del Balzo U, et al. Ischemia-reperfusion injury at the microvascular level: treatment by endothelin-a selective antagonist and evaluation by myocardial contrast echocardiography. Circulation 2000;102:3111-6.

9 Gregorini L, Marco J, Kozakova M, et al. $\alpha$-Adrenergic blockade improves recovery of myocardial perfusion and function after coronary stenting in patients with acute myocardial infarction. Circulation 1999;99:482-90.

10 Skelding KA, Goldstein JA, Mehta L, et al. Resolution of refractory no-reflow with intracoronary epinephrine. Cathet Cardiovasc Intervent 2002;57:305-9.

11 Limbruno U, Micheli A, De Carlo M, et al. Mechanical prevention of distal embolization during primary angioplasty. safety, feasibility, and impact on myocardial reperfusion. Circulation 2003;108:171-6.

12 Stone GW, Brodie BR, Griffin JJ, et al, on behalf of the Primary Angioplasty in Myocardial Infarction (PAMI) Investigators. Improved short-term outcomes of primary coronary stenting compared to primary balloon angioplasty in acute myocardial infarction at experienced centers: the PAMI study group experience. J Intervent Cardiol 1999;12:101-8.

13 Neumann FJ, Blasini R, Schmitt C, et al. Effect of glycoprotein Ilb/Illa receptor blockade on recovery of coronary flow and left ventricular function after the placement of coronary artery stents in acute myocardial infarction. Circulation 1998;98:2695-701.

14 Ito H, Taniyama Y, Iwakura K, et al. Intravenous nicorandil can preserve microvascular integrity and myocardial viability in patients with reperfused anterior wall myocardial infarction. J Am Coll Cardiol 1999;33:654-60. 
15 Iwakura K, Ito $\mathrm{H}$, Ikushima $\mathrm{M}$, et al. Association between hyperglycemia and the no-reflow phenomenon in patients with acute myocardial infarction. J Am Coll Cardiol 2003;41:1-7.
16 Genda S, Miura T, Miki T, et al. K mechanism of protection against the no-reflow phenomenon but its function is compromised by hypercholesterolemia. J Am Coll Cardiol 2002;40:1339-46.

\section{IMAGES IN CARDIOLOGY}

\section{The paradoxical finding of mitral valve incompetence and cor triatriatum: a mechanism revealed?}

M agnetic resonance imaging demonstrated features of cor triatriatum in a 27 year old woman (upper panel). In the absence of associated congenital anomalies, obstructive cor triatriatum due to a small, flow limiting fenestration will present in a similar manner to mitral stenosis. However, this woman presented with exertional breathlessness, paroxysmal nocturnal dyspnoea, and prominent murmur of mitral incompetence.

Echo confirmed significant mitral regurgitation with an enlarged but contractile left ventricle. (End diastolic dimension $7 \mathrm{~cm}$ and end systolic dimension of $3.9 \mathrm{~cm}$.) Panels A-C below demonstrate the mechanism of association between cor triatriatum, mitral incompetence, and left ventricular dilatation. A fenestration jet impinges on the anterior mitral leaflet, holding it open at the onset of ventricular systole while the posterior leaflet is closing. This abnormality of phase over a chronic period appears to have led to a spiral of mitral incompetence and compensatory left ventricular dilatation. This hypothesis was confirmed by the result of the surgical intervention. With the aid of intraoperative transoesophageal echocardiograpy, it was elected to excise the membrane alone. The valve leaflets were neither repaired nor a ring inserted to tighten around the mitral valve annulus. Five weeks later echocardiography demonstrated that removal of the membrane had restored normal flow mechanics allowing the leaflets to move in phase, coapt, and for the valve to regain competence. Left ventricular end diastolic size had regressed to $5.1 \mathrm{~cm}$. No change in medication had occurred and the patient was symptom-free.

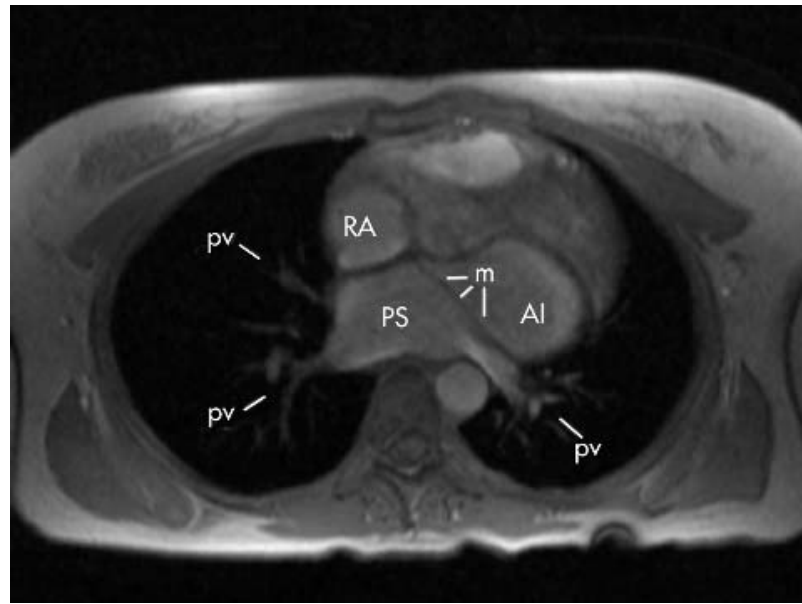

Transverse magnetic resonance image showing the "heart of three atria". Pulmonary veins enter the postero-superior chamber of the greatly enlarged left atrium. This is partitioned from the antero-inferior chamber by a fibromuscular membrane extending from below the fossa ovalis to the posterior free wall. Al, antero-inferior left atrial septum; m, membrane; PS, postero-superior left atrial chamber; pv, pulmonary veins; $R A$, right atrium.

W Keeble

E Lundmark H J Dargie williamkeeble@doctors.org.uk
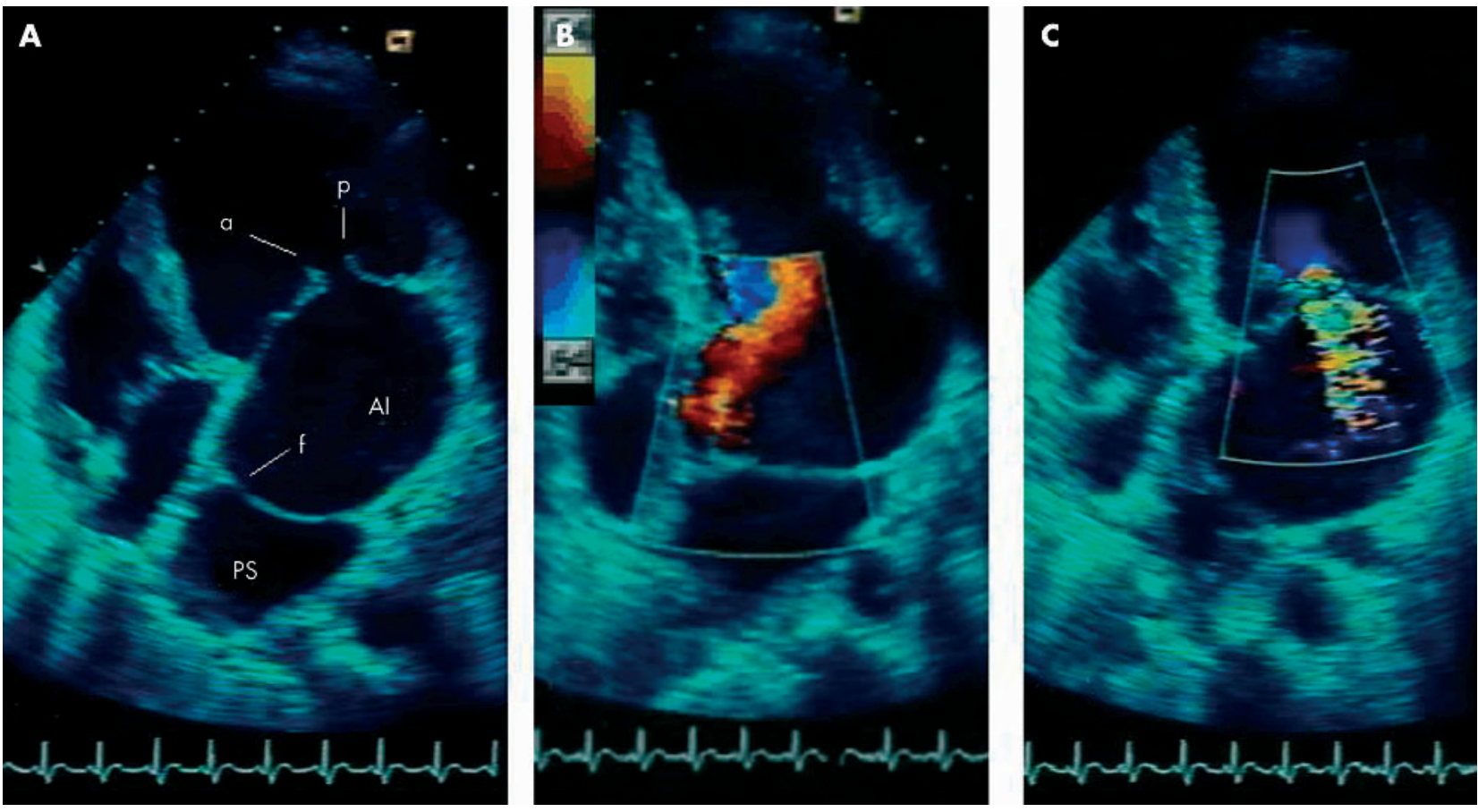

Transthoracic two dimensional echocardiography with colour flow mapping overlay reveals flow through the fenestration in the membrane (panel A). Flow extends along the interatrial septum to impinge upon the atrial side of the anterior mitral valve leatlet (panel B). A subsequent broad based jet of mitral regurgitation occurs (panel C). Al, antero-inferior left atrial septum; $a$, anterior mitral valve leaflet; $f$, fenestration; $p$, posterior mitral valve leaflet; PS, postero-superior left atrial chamber. 